\title{
New tricks for old JNKs
}

Although c-Jun N-terminal kinases (JNKs) are among the most abundant protein kinases in the brain, their physiological functions in neurons are yet to be established. Huganir and colleagues now identify two glutamate-receptor subunits as JNK substrates and show how JNKs regulate their trafficking.

Using a bioinformatics approach, the authors identified proteins that contain a consensus motif for phosphorylation by JNKs. Among these were two $\alpha$-amino-3-hydroxy5-methyl-4-isoxazole propionic acid (AMPA)-receptor subunits - GluR4 and the long splice form of GluR2 (GluR2L) - and in vitro analysis confirmed that these proteins could be phosphorylated by JNK1.

The GluR2L and GluR4 subunits displayed a high level of basal phosphorylation in both cultured cortical neurons and adult-rat brain extracts. Phosphorylation of both subunits was diminished by treatment with a JNK inhibitor or by overexpression of the JNK-binding domain of the scaffold protein JNK-interacting protein 1 (JIP1), which separates JNKs from their activators. JNK1, the principal JNK isoform that was found in the brain extracts, was also detected in a protein complex with GluR2L and GluR4, suggesting that JNK1 is responsible for maintaining these basal levels of phosphorylation.

The function of postsynaptic glutamate receptors can be modulated by phosphorylation in response to glutamatergic signalling, and the in vitro results suggested that JNKs might contribute to this regulation. Indeed, the authors showed that JNK1 and other JNK-pathway proteins are enriched in synaptic and postsynaptic density fractions. Furthermore, substances that enhance synaptic activity, such as NMDA ( $N$-methyl-D-aspartate), led to a rapid dephosphorlyation of both subunits in cultured neurons. Washout of NMDA returned the phosphorylation to basal levels.

As phosphorylation can affect glutamate-receptor trafficking, the authors created a modified, fluorescent form of GluR2L that loses fluorescence when it is internalized, allowing them to monitor its trafficking in live neurons. NMDA treatment caused rapid internalization of the subunit, followed by rapid re-insertion into the membrane upon NMDA washout. This re-insertion was reliant on JNK activity.

This study identified two endogenous substrates of JNKs in neurons and demonstrated a role for JNKs in the regulation of AMPA-receptor trafficking. As JNKs have been implicated in neurodegenerative disease, these findings might give us insights into how their dysfunction leads to neuropathology.

Katherine Whalley

ORIGINAL RESEARCH PAPER Thomas, G. M., Lin, D., Nuriya, M. \& Huganir, R. L. Rapid and bidirectional regulation of AMPA receptor phosphorylation and trafficking by JNK. EMBO J. 27, 361-372 (2008)

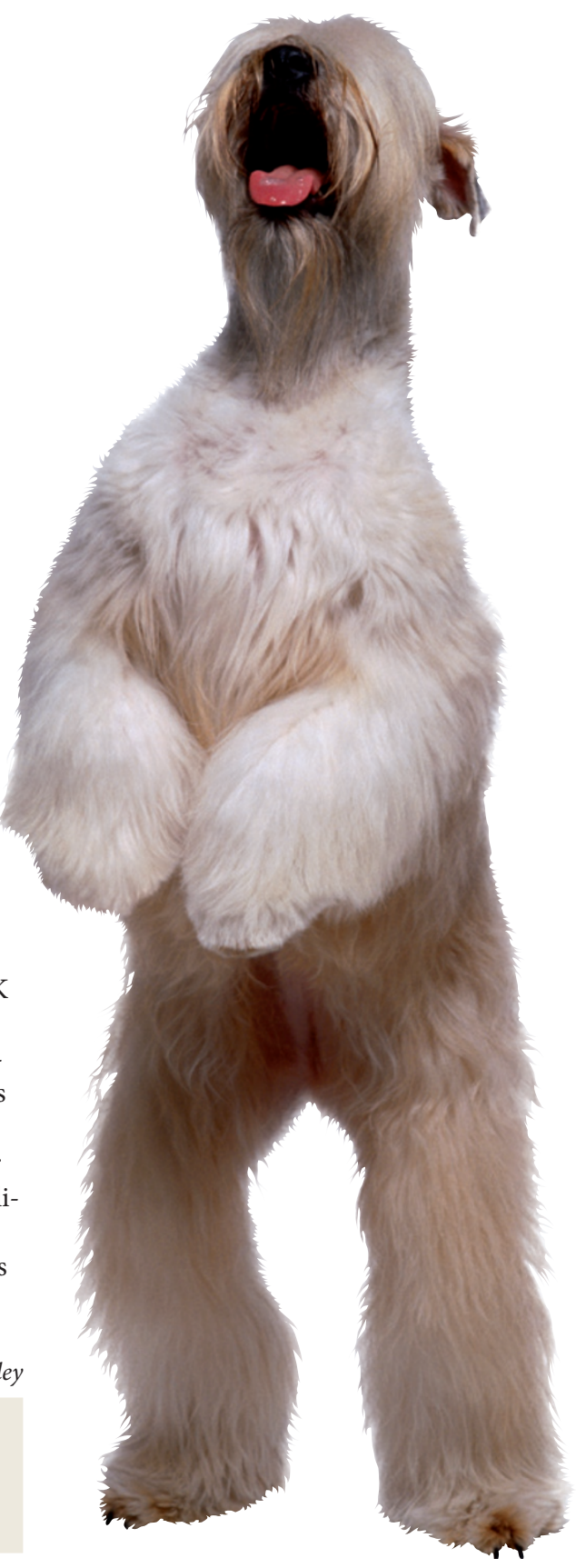

